NASA/TM- - 97ー 207858

$$
\begin{gathered}
\text { NM4-7ル } \\
119550
\end{gathered}
$$

\title{
Temperature Distribution in a Composite of Opaque and Semitransparent Spectral Layers R. Siegel
}

Reprinted from

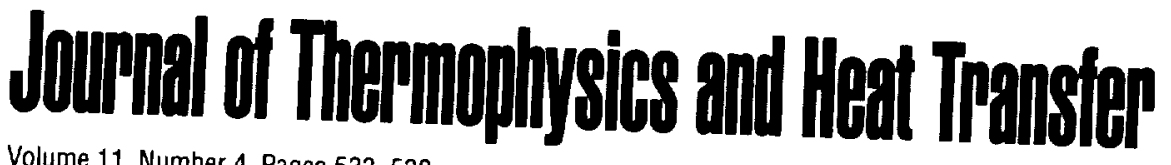

Volume 11, Number 4, Pages 533-539

\section{OAIAA}




\title{
Temperature Distribution in a Composite of Opaque and Semitransparent Spectral Layers
}

\author{
Robert Siegel* \\ NASA Lewis Research Center, Cleveland, Ohio 44135
}

\begin{abstract}
The analysis of radiative transfer becomes computationally complex for a composite when there are multiple layers and multiple spectral bands. A convenient analytical method is developed for combined radiation and conduction in a composite of alternating semitransparent and opaque layers. The semitransparent layers absorb, scatter, and emit radiation, and spectial propertic comparison with a basic solution included. The two-flux method is used, and its applicability is veriod solved by deriving a Green's function. in the literature. The differential equation in the two-nux method is solved by der zirconia thermal barrier The solution technique is applied to analyze radiation effects in a multiayer zustor. The zirconia radiative properties are modeled by two spectral bands. Thin opadating ability. With radiation shields, the decrease radiant transmission that can degrade the zirconia insulating abily. Wides the lowest metal wall temperature distributions more closely approach the opaque limit that prov temperatures.
\end{abstract}

\footnotetext{
Nomenclature

$a_{\lambda i}=$ spectral absorption coefficient of the $j$ th layer $\mathrm{m}^{-1}$

$B 0_{A,}, B 1_{\lambda,}=$ quantities $2\left(2-\varepsilon 0_{j}\right) / 3 \varepsilon 0_{j} K_{\Lambda}, D$, and $2\left(2-\varepsilon 1_{j}\right) / 3 \varepsilon 1, K_{\lambda} D_{j}$, respectively

$D$, = thickness of the $j$ th layer, $m$

$=$ blackbody spectral energy in vacuum,

$\mathrm{W} / \mathrm{m}^{2} \mu \mathrm{m} ; \tilde{e}_{\lambda b}=e_{\lambda b} / \sigma T_{g 1}^{4}$

$F_{\mathrm{l}}, F_{\mathrm{us}} \quad=$ fraction of blackbody energy from $\lambda=0$ to the lower or upper wavelength limit of the $s$ spectral band

$G_{\lambda}=$ two-flux spectral flux quantity, $W / \mathrm{m}^{2} \mu \mathrm{m}$; $\tilde{G}_{\lambda}=G_{\lambda} / \sigma T_{g 1}^{4}$

$g_{\lambda}\left(X_{j}\right)=$ Green's function for $\bar{G}_{\lambda}\left(X_{j}\right)$ in semitransparent $j$ th layer

= convective heat transfer coefficients at external boundaries, $\mathrm{W} / \mathrm{m}^{2} \mathrm{~K} ; H=h / \sigma T_{k 1}^{3}$

$K_{\lambda_{j}} \quad=$ spectral extinction coefficient of the

$K_{\lambda j} \quad=$ spectral extinction cerfer, $a_{\lambda j}+\sigma_{s \lambda j}, \mathrm{~m}^{-}$

$k \quad=$ thermal conductivity of the $j$ th layer, $\mathrm{W} / \mathrm{m} \mathrm{K}$; $N_{1}=k_{i} / \sigma T_{* 1}^{3} D_{j}$

$m_{\lambda ;}=$ quantity $K_{\lambda,} D_{1}\left[3\left(1-\Omega_{\lambda, j}\right)\right]^{1 / 2}$

$m_{\lambda,} \quad=$ refractive index of semitransparent material

$n \quad=$ heat flux. $\mathrm{W} / \mathrm{m}^{2}: \tilde{q}=q / \sigma T^{4}$

.

$y_{\text {to }}=$ total heat flux by

$q_{x+1}, q_{r_{2}}=$ radiant spectral energy incident at hot and cold

$q_{x+1} \cdot q_{\times r 2}=$ sides, $W / \mathrm{m}^{2} \mu \mathrm{m}$. Fig. 1

$T_{k 1}, T_{Q 2}=$ gas temperatures on hot and cold sides of composite. K. Fig. 1

$T \quad=$ absolute temperature in $j$ th layer, $\mathrm{K} ; t_{j}=T_{j} / T_{k}$

$T_{1}, T_{1}=$ effective blackbody temperatures of surroundings outside of hot and cold sides, $\mathrm{K}$

- 1997: revivion received April 4, 1997: accepted Received Feb. 14. 1997: revision received April 4, 1997 by the American for publication April 4, 1997. Copyright (C) No copyright is asserted Institute of Acronautics and Astronatics Code The U.S. Government in the United States under Title has a royalty-free license to exercise all rights under rights are reclaimed herein for Governmental

served by the copyright owner.

*Senior Research Scientist, Researc
21000 Brookpark Road. Fellow AlAA
}

$x_{j}=$ coordinate in $j$ th layer, m. Fig. la; $X_{j}=x_{j} / D$,

$\quad=$ emissivity of opaque metal barrier (radiation shield)

= emissivities of opaque materials (as measured in environment with $n=1$ ) at left and right boundaries of semitransparent $j$ th layer, Fig. 2

$=$ emissivities at external boundaries, Fig. 1

$\varepsilon_{03}, \varepsilon_{o 2} \quad=$ optical thickness of $j$ th layer in the $s$

$\kappa_{\mathrm{sj}} \quad$ wavelength band, $K_{\mathrm{ij}} D_{j}$

$\lambda \quad=$ wavelength of radiation, $\mu \mathrm{m}$

$=$ integration coordinate in Green's function

$\xi \quad=$ Stefan-Boltzmann constant, $\mathrm{W} / \mathrm{m}^{2} \mathrm{~K}^{4}$

$=$ scattering coefficient in the semitransparent $j$ th layer, $\mathrm{m}^{-1}$

$=$ scattering albedo in semitransparent $j$ th layer. $\sigma_{s \lambda} / K_{\lambda j}$

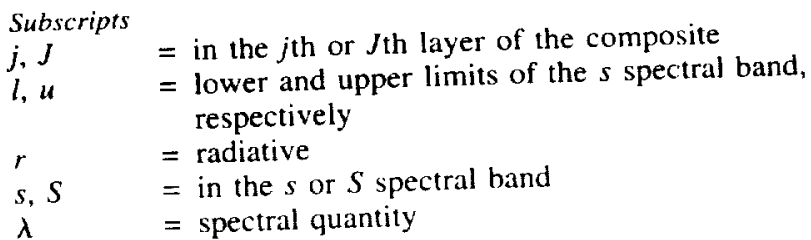

Superscript

$\sim \quad$ dimensionless quantity

\section{Introduction}

A $\mathrm{N}$ analytical method is developed to determine heat transfer in a multilayer composite where alternate layers are semitransparent. Computations for a composite can become complex when many layers are involved and they have spectral property variations. The method is applied to examine the heat transfer characteristics of thermal barrier coatings.

Thermal barrier coatings are important for many high-temperature applications, including combustor liners, turbine vanes, and rotating blades for current and advanced aircraft engines. Some of the insulating materials used for coatings, such as zirconia, which currently has widespread use, are partially transparent to thermal radiation. ${ }^{1-3}$ Internal radiative transfer effects are increased as temperatures are raised to obtain higher engine efficiencies. The radiative transfer can act 
as an apparent increase in thermal conductivity that can degrade the insulating material effectiveness. It is necessary to quantitatively estimate the radiation effect, and if necessary, provide a means for reducing radiative transfer.

A possible reduction method is to place opaque barriers within the coating to act as radiation shields, such as thin metal foils with a high melting temperature. The result is a composite of alternating semitransparent and opaque layers (Fig. 1). As stated earlier, the computations become more difficult as the number of layers is increased, and also when spectral bands must be included, as for zirconia. This paper will develop a convenient analytical procedure for obtaining the temperature distribution and heat flow within such a composite. The method can include large scattering in the semitransparent layers, which is typical of a thermal protection material such as zirconia. Results including radiation within the semitransparent insulating material are compared with heat conduction calculations with internal radiation neglected; in this limiting case, radiant absorption and emission occur only at the external surfaces.

Internal radiation effects in a thermal barrier coating were demonstrated in Ref. 5, where an analytical procedure was developed using the two-flux equations for the radiative contribution. The two-flux method includes, without difficulty, the large scattering characteristic of a material such as zirconia The analysis in Ref. 5 considered two spectral regions, one semitransparent and the other opaque, that approximated zirconia transmission behavior.' The analysis was improved in Ref. 6 to incorporate a wider range of spectral property variations and optical thicknesses. This was done by deriving a Green's function that provides an analytical solution for the two-flux differential equation. The analytical relations incorporate the two-point boundary conditions that must be satisfied for the solution in a semitransparent layer, and this simplifies obtaining a converged iterative solution for the temperature distribution. A convergence procedure was provided that works well.

The development of a Green's function for a single layer ${ }^{6.7}$ is expanded here for use in multiple layers, as in Fig. 1, where each semitransparent layer has opaque boundaries. As demonstrated for several cases in Ref. 8, the two-flux equations

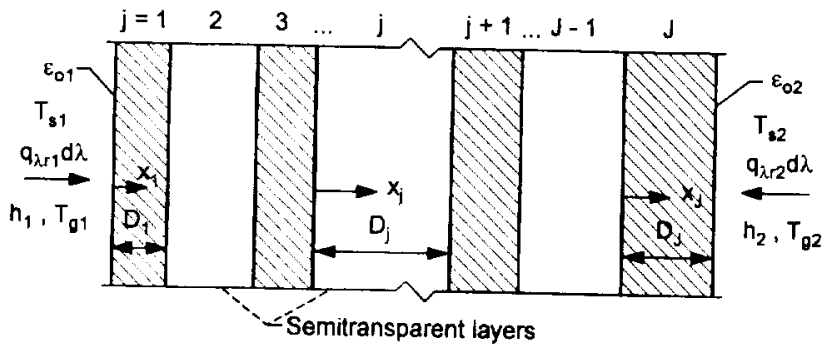

a)

(Opaque layers are shaded)

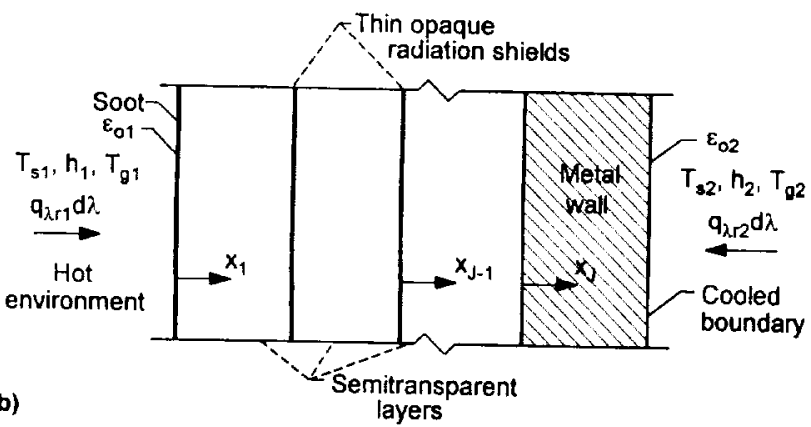

Fig. 1 Geometry and nomenclature for a multilayer composite of alternate semitransparent and opaque layers with external radiation and convection at both exposed surfaces: a) nomenclature for a general series of layers and $b$ ) a soot-covered thermal barrier coating on a metal wall, with internal opaque radiation shields. provide accurate results for single spectral layers with diffuse boundaries, as assumed here. Hence, the two-flux method is used to provide a simplified procedure, because the exact spectral radiative transfer equations including large scattering become rather complicated for multiple layers with spectral properties. The analytical expressions provide the temperature distribution and heat flow in a composite of alternate semitransparent and opaque layers. Results are given for the reduction in radiative effects in a thermal barrier coating on a combustor liner. when shields are introduced within the semitransparent insulation (Fig. 1b). The outside of the coating exposed to the hot environment has an opaque layer of soot. The cooled side of the coating is bounded by the metal wall of the combustor.

\section{Analysis}

A composite consists of alternate semitransparent and opaque layers (Fig. la). An example is a multilayer thermal barrier coating on a metal wall, where successive semitransparent insulating layers are bounded by internal opaque metal radiation shields (Fig. 1b). In each semitransparent layer, the total heat flux is the sum of radiation and conduction; from energy conservation, the total flux is constant in the $x$ direction. Heat flows through the opaque layers by conduction only. The temperature distribution in each semitransparent layer can be obtained by integrating the energy equation, to give $e^{5,6}$

$$
T_{j}\left(x_{j}\right)=T\left(x_{j}=0\right)-\frac{1}{k_{j}}\left[q_{1001} x_{j}-\frac{1}{3} \int_{\lambda=0}^{\infty} \frac{G_{\lambda}\left(x_{j}=0\right)-G_{\lambda}\left(x_{j}\right)}{K_{\lambda j}} \mathrm{~d} \lambda\right]
$$

To obtain the temperature distribution from this relation, the $q_{\mathrm{to}}$, and the $T\left(x_{j}=0\right)$ and $G_{\lambda}\left(x_{j}\right)$ for each layer, must be determined as will be described.

The flux quantity $G_{\lambda}\left(x_{j}\right)$ in each semitransparent layer is obtained by solving a second-order differential equation, ${ }^{5}$ that includes the local blackbody emission, $e_{A b}(x)$

$$
\frac{\mathrm{d}^{2} G_{\lambda}\left(x_{j}\right)}{\mathrm{d} x_{j}^{2}}-3 K_{\lambda j}^{2}\left(1-\Omega_{\lambda j}\right) G_{\lambda}\left(x_{j}\right)=-3 K_{\lambda j}^{2}\left(1-\Omega_{\lambda j}\right) 4 n_{j}^{2} e_{\lambda b}\left(x_{j}\right)
$$

The $e_{\lambda b}\left(x_{j}\right)$ is obtained by iteration during the solution, since the temperature distribution is unknown.

Two boundary conditions are required for solving Eq. (2) in each semitransparent layer. At $x_{y}=0$, in Fig. 2, the adjacent opaque barrier has an emissivity $\varepsilon 0_{j}$. Note that tabulated emissivity values are for emission into surroundings with a refractive index of one; for emission into a material with refractive index $n_{j}$, a multiplying factor $n_{j}^{2}$ must be included. Then, the boundary condition for $G_{\lambda}\left(x_{j}=0\right)$, at the left bound-

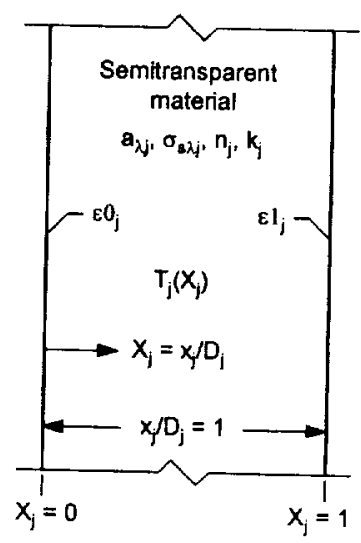

Fig. 2 Geometry and boundary emissivities for a single semitransparent layer between opaque walls; this is a basic unit for the Green's function solution. 
ary of the semitransparent $j$ th layer (Fig. 2), in the composite coating is

$$
G_{\lambda}\left(x_{j}=0\right)-\left.\frac{2}{3 K_{\lambda j}}\left(\frac{2}{\varepsilon 0}-1\right) \frac{\mathrm{d} G_{\lambda}}{\mathrm{d} x}\right|_{l_{j}=0}=4 n_{j}^{2} e_{\lambda b}\left(x_{j}=0\right)
$$

At the right side of the $j$ th layer in Fig. $2, x_{j}=D_{j}$, the emissivity of the opaque boundary (for emission into surroundings with a refractive index of one) is $\varepsilon l_{j}$. The boundary condition is then'

$$
G_{A}\left(x_{j}=D_{j}\right)+\left.\frac{2}{3 K_{\lambda}}\left(\frac{2}{\varepsilon 1_{j}}-1\right) \frac{\mathrm{d} G_{\lambda}}{\mathrm{d} x}\right|_{\lambda_{j}=D_{j}}=4 n_{j}^{2} e_{\lambda b}\left(x_{j}=D_{j}\right)
$$

A solution for Eq. (2), in analytical form. that satisfies the boundary conditions Eqs. ( $3 a$ ) and (3b) is obtained by deriving a Green's function. Following the procedure in Ref. 7 (that had different boundary conditions), the Green's function in terms of dimensionless variables is derived as condition of a partially transmitting surface can be included, as given in Ref. 6. For a thermal barrier coating, an opaque boundary can be produced by the semitransparent material having a soot coating as in a combustor; this is shown at $x_{1}=$ 0 in Fig. 1b. At the cooled side of the composite, the semitransparent coating is on an opaque metal wall (Fig. 1b). The total heat flux at the opaque surface $x_{1}=0$, consists of convection and radiation exchange. The spectral radiation flux incident from the external surroundings is $q_{\lambda r 1} \mathrm{~d} \lambda$, and so the heat balance becomes. with the assumption that the surface at $x_{1}=0$ is gray

$$
q_{1+1)}=h_{1}\left[T_{g 1}-T_{1}\left(x_{1}=0\right)\right]+\varepsilon_{o 1}\left[q_{r 1}-\sigma T_{1}\left(x_{1}=0\right)^{4}\right]
$$

where $q_{r}$ is the incident radiation flux including energy at all wavelengths. A similar relation is written for the external boundary at the right side of the composite in Fig. 1:

$$
q_{101}=h_{2}\left[T_{J}\left(x_{J}=D_{j}\right)-T_{R^{2}}\right]+\varepsilon_{\omega 2}\left[\sigma T_{J}\left(x_{J}=D_{J}\right)^{4}-q_{r 2}\right]
$$

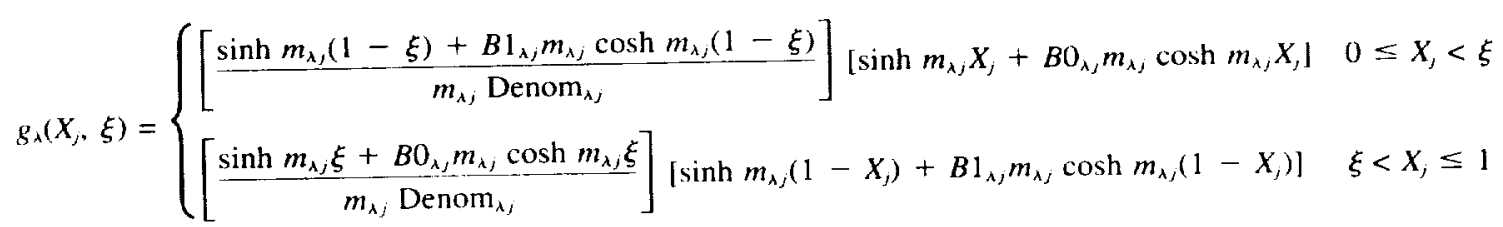

where $X_{i}=x_{i} / D_{i}$, and the denominator in Eq. (4a) is

$$
\begin{gathered}
\text { Denom }_{\lambda,}=\left(1+B 0_{\lambda} B 1_{\lambda j} m_{\lambda}^{2}\right) \sinh m_{\lambda} \\
+\left(B 0_{\lambda j}+B 1_{\lambda}\right) m_{\lambda j} \cosh m_{\lambda j}
\end{gathered}
$$

The Green's function $g_{\lambda}\left(X_{j}, \xi\right)$ is used to account for the nonhomogeneous term in Eq. (2), when computing the solution for $\tilde{G}_{\lambda}\left(X_{j}\right)$. To obtain the complete solution for $\tilde{G}_{A}\left(X_{j}\right)$, the solution is needed for the homogeneous part of Eq. (2). Following the procedure in Ref. 7, this solution is

$$
\tilde{G}_{h \lambda}(X)=C_{\lambda j} \sinh m_{\lambda j} X_{j}+D_{\lambda}, \cosh m_{\lambda j} X
$$

The boundary conditions, Eqs. (3a) and (3b), are applied to give the following relations to evaluate $D_{\lambda j}$, and then $C_{\lambda j}$ :

$$
\begin{gathered}
D_{\lambda j}=\frac{4}{\operatorname{Denom}_{\lambda j}}\left\{\left(\sinh m_{\lambda j}+B 1_{\lambda j} m_{\lambda}, \cosh m_{\lambda j}\right)\right. \\
\left.\times n ; \tilde{e}_{\lambda b}\left(X_{j}=0\right)+B 0_{\lambda j} m_{\lambda,} n_{j}^{2} \tilde{e}_{\lambda b}\left(X_{j}=1\right)\right] \\
C_{\lambda j}=\frac{D_{\lambda j}-4 n_{j}^{2} \tilde{e}_{\lambda \nu}\left(X_{j}=0\right)}{B 0_{\lambda j} m_{\lambda j}}
\end{gathered}
$$

By adding $\tilde{G}_{h A}(X)$ from Eq. (5) to the nonhomogeneous solution obtained by using the Green's function. $g_{A}\left(X_{j}\right)$, the solution of Eq. (2) is

$$
\tilde{G}_{\lambda}(X)=\tilde{G}_{h_{A}}\left(X_{j}\right)+4 m_{\lambda}^{2} n_{j}^{2} \int_{0}^{1} g_{\lambda}\left(X_{j}, \xi\right) \tilde{e}_{\lambda b}\left(X_{j}=\xi\right) \mathrm{d} \xi
$$

Now that the solution for $G_{\lambda}\left(x_{j}\right)$ has been obtained, relations will be found for $T\left(x_{j}=0\right)$ for each semitransparent layer, and for $q_{t w 1}$, as needed to evaluate Eq. (1) for the semitransparent layers. These quantities are obtained by writing a total energy flux relation for each layer in the composite.

At each outer boundary of the composite in Fig. la or 1b, it is assumed that there is either an opaque coating or layer, so that radiation from the surroundings cannot be transmited through the surface. For a clean semitransparent boundary, the
For an opaque material, such as a metal wall, heat is transferred only by conduction, and so by using the temperature difference across any opaque layer of finite thickness in Fig. la or $1 \mathrm{~b}$

$$
q_{\mathrm{tot}}=\left(k_{j} / D_{j}\right)\left[T_{j}\left(x_{j}=0\right)-T_{j}\left(x_{j}=D_{j}\right)\right]
$$

If there is a very thin opaque barrier between the $j$ and $j+1$ semitransparent layers, it is assumed that there is negligible temperature change across the barrier, and so the continuity of temperature gives $T_{,}\left(x_{j}=D_{j}\right)=T_{j+1}\left(x_{j+1}=0\right)$.

For each semitransparent layer, the total heat flow is given by Eq. (1) as

$$
\begin{aligned}
q_{101} & =\frac{k_{j}}{D_{j}}\left[T_{j}\left(x_{j}=0\right)-T_{j}\left(x_{j}=D_{j}\right)\right] \\
& +\frac{1}{3 D} \int_{\text {semirran-pareat vectral hands }} \frac{G_{\lambda j}\left(x_{j}=0\right)-G_{\lambda j}\left(x=D_{j}\right)}{K_{\lambda j}} \mathrm{~d} \lambda
\end{aligned}
$$

Equations (7) are written for each external boundary and for each layer. If the $G_{\lambda}\left(x_{j}\right)$ are known, as will be obtained in the iterative solution, this provides a set of simultaneous equations that can be solved numerically for $q_{10 t}$ and for the temperature at each interface. The temperature distribution in each layer is then found by using Eq. (1), with the integral term omitted for an opaque layer. The converged solution for $T(x)$ in all of the layers is found by iteration, as will be described.

\section{Multiple Spectral Band Form of the Radiative Relations}

For a material like zirconia, that is currently used for turbine engine thermal barrier coatings, the semitransparent spectral region extends to a cutoff wavelength of about $5 \mu \mathrm{m}$. beyond which the extinction coefficient becomes quite large and the coating is usually assumed opaque. ${ }^{1.9}$ Multiple spectral bands for zirconia or other semitransparent insulating materials can be used to include spectral property variations in the semitransparent region. The relations are given here for $S$ spectral bands $(s=1 \ldots, S)$, in the region $\lambda=0$ to $\lambda_{\mathrm{us}}$, where $\lambda_{\mathrm{us}}$ is at the 
upper limit of the $S$ band, and the coating is opaque in the range $\lambda>\lambda_{\text {us }}$ above the upper limit of the $S$ band.

To obtain the temperature distribution in each semitransparent layer, Eq. (1) is summed over the $s$ bands from $s=1$ to $S$ in the semitransparent region, to give in dimensionless form

$$
t_{j}\left(X_{j}\right)=t_{j}\left(X_{j}=0\right)-\frac{1}{N_{j}}\left[\tilde{q}_{\mathrm{tot}} X_{j}-\frac{1}{3} \sum_{s=1}^{s} \frac{\tilde{G}_{s j}\left(X_{j}=0\right)-\tilde{G}_{s j}\left(X_{j}\right)}{\kappa_{s j}}\right]
$$

To obtain $\tilde{G}_{s j}\left(X_{j}\right)$, Eq. (6) is used for each band in the form

$$
\begin{aligned}
& \tilde{G}_{\mathrm{s},}(X)=\tilde{G}_{h s}(X)+4 m_{\mathrm{s} j}^{2} n_{j}^{2} \int_{0}^{1} g_{s j}(X, \xi) t,(\xi)^{4}\left\{F_{\mathrm{us}}[t,(\xi)]\right. \\
& \left.\quad-F_{\mathrm{ls}}[t,(\xi)]\right\} \mathrm{d} \xi
\end{aligned}
$$

where $\tilde{G}_{h s}\left(X_{j}\right)$ and $g_{\mathrm{v} j}$ are evaluated using the properties in the $s$ band. The $F_{\text {us }}(t)$ is the fraction of blackbody energy for emission at temperature $t$, in the wavelength range $\lambda=0$ to $\lambda_{u s}$, where $\lambda_{\text {us }}$ is at the upper limit of the $s$ band, and $F_{\text {is }}$ corresponds to the lower limit of the band, $\lambda=\lambda_{\text {ss }}$. The $F(t)$ was evaluated from the summation form in Ref. 9. The band form of Eq. (7d) is

$$
\begin{aligned}
\tilde{q}_{\mathrm{tot}} & =N_{j}\left[t_{j}\left(X_{j}=0\right)-t,\left(X_{j}=1\right)\right] \\
& +\frac{1}{3} \sum_{s=1}^{s} \frac{\tilde{G}_{s j}\left(X_{j}=0\right)-\tilde{G}_{s j}\left(X_{j}=1\right)}{\kappa_{s j}}
\end{aligned}
$$

\section{Solution Method}

An iterative solution was used to obtain $t(X)$ in all of the layers, and it was found to give rapid convergence for the calculations made here for the engine environment. Less than 20 iterations were usually required to reach convergence to five figures in the dimensionless temperature. For parameters typical of an engine, the heat transfer depends more on conduction than radiation. For other situations, where radiation is more dominant, the convergence rate can be considerably reduced, and it is usually necessary to use a damping factor to provide a small change between iterations and to run hundreds of iterations. Because convection and conduction are more important than radiation in an engine environment, the heat conduction solution, with all layers assumed opaque, was used as a first guess for the temperature distribution throughout the composite. Then, using the radiation properties for each spectral band, the Green's function was evaluated from Eq. (4) for each spectral band in each semitransparent layer, and the homogeneous solution in each layer was evaluated from $\mathrm{Eq} .(5)$. Double precision was used for all evaluations. Using Eq. (9) (evaluated by Romberg integration using a standard computer subroutine), then gave the two-flux quantity $\tilde{G}_{s j}\left(X_{j}\right)$ for each semitransparent layer and spectral band. Equations (7), written for each external interface and for each layer, were then solved numerically for $\tilde{q}_{w t}$, the $t_{j}(0)$ for each layer, and $t,(1)$; a computer subroutine was used based on Newton's method. Then, Eq. (8) was used to evaluate the temperature distribution $t_{j}\left(X_{j}\right)$ in each semitransparent layer. These temperature distributions were used to start the next iteration using Eqs. (4) and (5), and the procedure was continued until $t_{j}\left(X_{j}\right)$ converged. As discussed earlier, convergence was rapid for an engine environment because the final solutions are fairly near the solutions with only heat conduction, and so it is a good approximation to assume opaque layers to begin the iteration. The accuracy of typical results was checked using 21.41 , and $81 X_{i}$ points.

To further examine the basic solution method. a radiationdominated case was evaluated and compared with the results of an exact solution in the literature, for a layer between two opaque walls at specified temperatures. The solution for conduction, as the only mode of transfer, was used to start the calculation to investigate convergence when radiation is dominating. It was necessary for stability to use a damping factor of 0.05 to reduce the temperature change between iterations. and about 100 iterations were required for convergence. Good agreement was obtained with the exact solution, as will be shown.

\section{Results and Discussion}

Some illustrative results will be given for the behavior of thermal barrier coatings with and without internal radiation shields in the environment of a turbine engine combustion chamber. First, however, a comparison is made of results from the present method with a fundamental solution in the literature. The additional effect of internal radiation shields will be shown for the fundamental boundary conditions of a layer between black walls at specified temperatures. The behavior is found to be quite different from that in an engine environment with convective and radiative boundary conditions.

Results are given in Ref. 10 for temperature distributions by combined radiation and conduction in a gray medium between black opaque walls. The Green's function developed here applies for that situation. The case selected for comparison is shown in Fig. 3a; the semitransparent layer is between black
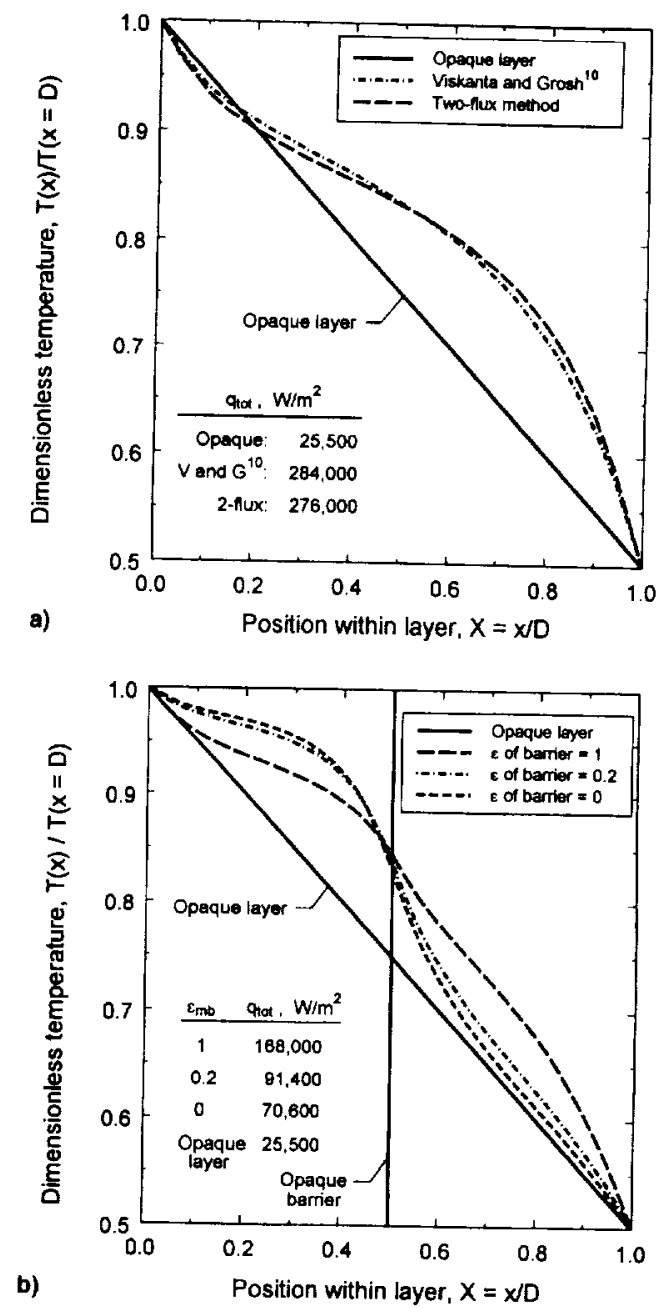

Fig. 3 Comparison with the classical solution of a semitransparent layer between two black opaque walls, and the effect an internal opaque shield. Parameters: $T(0)=1666.7 \mathrm{~K}, T(D)=833.3$ $\mathrm{K}, N=k a / 4 \sigma T(0)^{3}=0.02916:$ a) comparisons of the present twoflux Green's function solution with an exact solution, 10 using the radiative transfer equations (this solution is dominated by radiation) and b) the effect of an internal opaque radiation shield with emissivities $\varepsilon_{\mathrm{mb}}=0,0.2$, and 1 . 
walls with the specified temperature of the cooler wall equal to one-half the temperature of the hotter wall. The results are for a small value of the conduction-radiation parameter, $N=$ $k a / 4 \sigma T(0)^{3}=0.02916$, using the definition of $N$ in Ref. 10 . For these conditions, radiation has a large influence, and the $q_{\text {wx }}$ values in Fig. 3a are much larger when radiation is included. This is in contrast with a turbine engine environment, where conduction and convection effects are more dominating, and the conduction-radiation parameter is much larger. As shown on Fig. $3 a$, the temperatures and heat flux results from the two-flux solution are in good agreement with the solution from Ref. 10, where the equations of radiative transfer were solved for an absorbing material without scattering. This comparison indicates that the results that follow for a combustor should be accurate, because they have a smaller effect of radiation; it is the radiative term that is being approximated by the two-flux assumption. Other comparisons of two-flux and exact solutions are shown in Ref. 8 , for layers with boundaries that are partially transmitting rather than opaque, as in the present analysis.

As an example of applying the present solution method for multiple layers, Fig. $3 \mathrm{~b}$ shows the effect on the temperature distribution and heat flux by introducing an opaque barrier (radiation shield) at the center of the layer in Fig. 3a. Results are given for three emissivities on both sides of the barrier, $\varepsilon_{\mathrm{mb}}=1.0,0.2$, and the limit $\varepsilon_{\mathrm{mb}} \rightarrow 0$ (perfectly reflecting barrier). A barrier prevents the direct transmission of radiation across the entire layer, but radiation is still transmitted within each half of the layer and has a significant effect on the temperature distribution. Even a black radiation shield has a considerable effect, as is evident by comparison with the unobstructed layer in Fig. 3a. At the black barrier there is radiative absorption and re-emission, and the net radiative heat flow must be carried through by heat conduction. The $q_{\text {to }}$ values in Fig. 3 show that the energy flow is considerably reduced by the shield for this situation, where radiation is large compared with conduction. Since radiation cannot be transmitted through the shield, emission from the high-temperature boundary at $x=0$ is concentrated more within the left half of the layer, and its temperatures are raised; whereas the temperatures in the right half decrease (compare with Fig. 3a). As the barrier emissivity is decreased and, hence, reflected energy is increased, the temperatures in the cooler half of the layer are closer to the line for an opaque layer, where there is only heat conduction. Reflections from the barrier retain energy in the hotter side of the layer, where temperatures are increased. The total heat flux with a perfectly reflecting barrier is about three times that for an opaque layer, as a result of radiative transfer within the semitransparent material.

The method developed here is now applied for a thermal barrier coating. Results in Fig. 4 are for typical conditions in an aircraft engine combustor. The geometry is like that in Fig. lb. For Fig. 4a, an alloy steel combustor liner, $0.794 \mathrm{~mm}$ thick. is protected by a 1.0 -mm-thick zirconia coating. For an advanced engine, the combustion chamber pressure is high enough that the combined gas and soot radiation are assumed to provide a black environment at $T_{\lrcorner 1}=T_{81}$. This specifies the spectral (blackbody) distribution of the incident radiation. As is likely in practice, the exposed surface of the coating is assumed to be covered with soot. This provides a thin opaque coating with an emissivity assumed as $\varepsilon_{\text {soor }}=0.97$ (soot reflectivity $=0.03$ ). Energy is absorbed by the soot layer and is reradiated into the surroundings and the zirconia coating. The zirconia spectral radiation properties were approximated from Ref. 1 as having a semitransparent band at wavelengths $\lambda=$ $0-5 \mu \mathrm{m}$, with absorption and scattering coefficients, $a_{\lambda}=30$ $\mathrm{m}^{-1}$ and $\sigma_{s \lambda}=10,000 \mathrm{~m}^{-1}$, respectively; the zirconia is thus a highly scattering material. The zirconia is assumed opaque for $\lambda>5 \mu \mathrm{m}$. The effective blackbody surroundings temperatures and the convection parameters are in the caption of Fig. 4. At the cooled side of the composite, it is assumed that
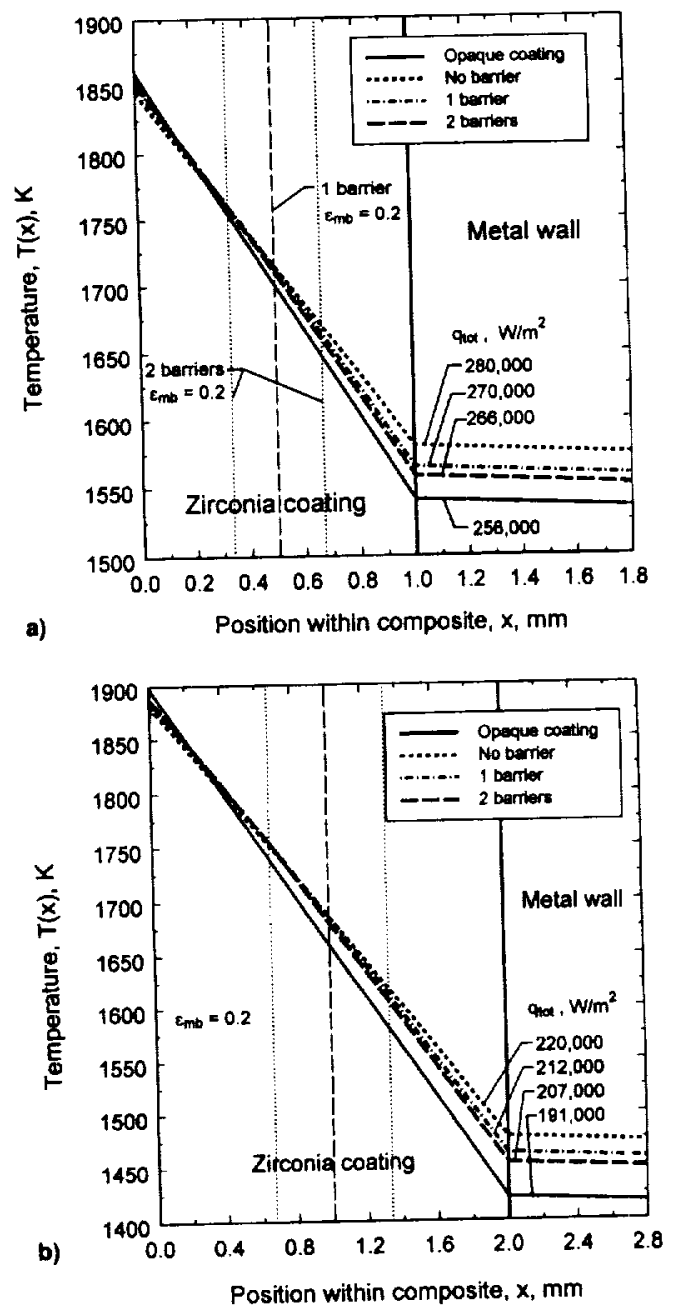

Fig. 4 Temperature distributions in a zirconia thermal barrier Fig. 4 Temperature dist a combustor, compared with an opaque thermal barrier coating. The effect of 1 or 2 internal radiation thermal barrier coating. The emissivity of 0.20 . Parameters (units shields is shown for a shield emissivity of 0.20 . Parameters (unt $=0.794 \cdot 10^{-3}, n=1.58, T_{s 1}=T_{g 1}=2000$, and $T_{p 2}=800$; for semi$=0.794 \cdot 10^{-3}, n=1.58, T_{s 1}=T_{s 1}=2000,10,000$ for $\lambda=0-5 \mu \mathrm{m}$; and the coating is opaque for $\lambda>5 \mu \mathrm{m}$ : thickness of zirconia coating, a) 1 and b) $2 \mathrm{~mm}$.

the metal surface is radiating into large blackbody surroundings at $T_{12}=T_{82}$, in addition to being convectively cooled. The cooled surface is assumed oxidized with an emissivity of 0.6 . The internal surface of the metal wall that is in contact with the coating is assumed to be clean, with an emissivity of 0.3 .

The solid line in Fig. 4a that has the lowest temperatures in the metal wall is for the limit where internal radiation is neglected and, hence, the coating is opaque; there is radiative exchange only at the coating surface. When the coating is partially transparent, internal radiant emission and transmission increase the energy transfer ability within the coating. This can be thought of as an increase in an effective thermal conductivity of the coating. It produces increased metal temperatures compared with an opaque coating; $40 \mathrm{~K}$ higher for this example for a coating without an internal radiation shield (upper dotted line in Fig. 4a).

When an internal shield with an emissivity of $\varepsilon_{\mathrm{mb}}=0.20$ is placed at the center plane of the coating, the radiative transfer is reduced, and the temperatures (dot-dash line) are closer to the opaque limit; the metal temperature is reduced relative to the coating without a barrier, and is now $24 \mathrm{~K}$ above the opaque limit. The addition of a second barrier, so that the 
coating is divided into three equal parts, provides a further reduction in metal temperature of $7 \mathrm{~K}$. The very thin shields must be made of a metal with a higher melting temperature than the alloy steel wall.

If possible, a thicker zirconia coating could be used in a combustor to reduce the metal temperatures. As an illustration of the effect of coating thickness, results for a coating $2 \mathrm{~mm}$ thick are in Fig. $4 \mathrm{~b}$. The metal temperatures are considerably reduced by the increased insulation thickness, but the effect of semitransparency remains similar to that in Fig. 4a. Without any internal radiation barrier, the metal wall temperature is increased about $56 \mathrm{~K}$, as a result of partial transparency of the coating. With two radiation shields having emissivities of 0.20 , the effect of radiation is reduced, so that the metal wall temperature is about $32 \mathrm{~K}$ above the opaque limit. Further decreases can be obtained if it is possible to reduce the emissivity of the shields as shown in Fig. 5.

So that the temperature scale can be expanded, only the temperatures in the metal wall are shown in Fig. 5. In Fig. 5a, for a coating thickness of $1 \mathrm{~mm}$, metal temperatures are included for the results in Fig, $4 \mathrm{a}$, with $\varepsilon_{\mathrm{mb}}=0.20$, and two lines are added that are closer to the opaque limit. These are for one and two barriers having an emissivity of $\varepsilon_{\mathrm{mb}}=0.05$. This illustrates the effect of a smaller shield emissivity on reducing the metal wall temperatures. At elevated temperatures, how-
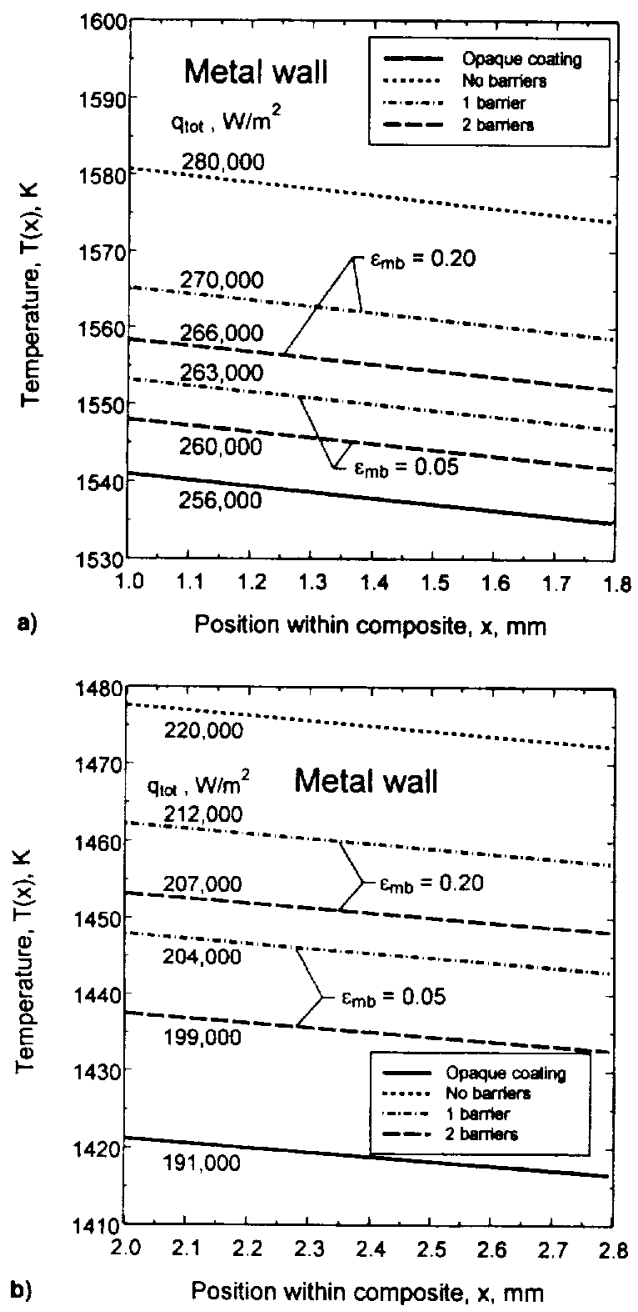

Fig. 5 Temperature distributions in the metal wall for a semitransparent thermal barrier coating on the wall of a combustor, compared with an opaque thermal barrier coating. The effect of 1 or 2 internal shields is shown for shield emissivities of 0.05 and 0.20. Parameters are the same as for Fig. 4: thickness of zirconia coating, a) 1 and b) $2 \mathrm{~mm}$. ever, it may not be possible to have an emissivity this small. as metal emissivities increase with temperature.

Similar results are in Fig. 5b for a zirconia coating thickness of $2 \mathrm{~mm}$. The relative positions of the temperature lines and the effect of shield emissivity are the same, but the temperatures have been decreased by the increased coating thickness. The total heat fluxes in Fig. $5 \mathrm{~b}$ are somewhat reduced by the thicker zirconia layer compared with Fig. 5a. The radiation shields have a relatively small effect on the total heat flow because the heat flow is quite dependent on conduction and convection for combustor conditions.

\section{Concluding Remarks}

The solution for radiative transfer in multilayer composites can become complex when there are spectral property variations and scattering is large. A convenient solution method was developed to determine temperature distributions and heat flows for a composite composed of alternate semitransparent and opaque layers. As an example of its use, the analysis was applied to study the effect of internal radiation barriers (radiation shields) in a layer of semitransparent material. The solution was compared for verification, with a classical case in the literature for a layer between two black walls at specified temperatures, and the effect of internal radiation shields was illustrated. The method was then applied to examine radiative effects on temperature distributions in semitransparent zirconia thermal barrier coatings on a metal wall, and the effects of introducing opaque internal radiation barriers into the coating were shown.

The two-flux method was used in the radiative transfer anal$y$ sis. This can be readily applied for materials with high scattering, such as zirconia that is presently used for thermal barrier coatings. The two-flux differential equation was solved by obtaining a Green's function. This provided convenient analytical expressions that were incorporated into an iterative numerical solution for internal temperatures and the total heat flow.

Illustrative solutions for temperatures in zirconia thermal barrier coatings were obtained for parameters typical of a turbine engine combustor. A two-spectral-band approximation was used for the zirconia radiative properties. One radiation barrier was found to reduce by about one-half the effect on the metal wall temperature of internal radiation in the coating. The method can be readily applied to examine other ranges of parameters. The results for a combustor, where there are large effects of conduction and convection, were contrasted with those for a layer between parallel walls with large radiative transfer relative to conduction.

\section{References}

'Wahiduzzaman, S., and Morel, T., "Effect of Translucence of Engineering Ceramics on Heat Transfer in Diesel Engines," Oak Ridge National Lab. Rept., Sub/88-22042/2, Oak Ridge. TN, April 1992.

"Matthews, L. K., Viskanta, R., and Incropera, F. P., "Combined Conduction and Radiation Heat Transfer in Porous Materials Heated by Intense Solar Radiation," Journal of Solar Energy Engineering, Vol. 107, No. 1, 1985, pp. 29-34.

"Makino, T., Kunitomo, T., Sakai, J., and Kinoshita, H., "Thermal Radiative Properties of Ceramic Materials," Transactions of the Japan Sociery of Mechanical Engineers, Vol. 50, No. 452, 1984, pp. $1045-1052$

${ }^{4}$ Committee on Coatings for High-Temperature Structural Materials, "Coatings for High-Temperature Structural Materials," National Academy of Sciences, National Academy Press, Appendix B. Washington, DC, 1996, pp. 65, 66.

'Siegel, R., "Internal Radiation Effects in Zirconia Thermal Barrier Coatings," Journal of Thermophysics and Heat Transfer, Vol. 10. No. 4. 1996, pp. 707-709.

'Siegel, R., "Green's Function to Determine Temperature Distri- 
bution in a Semitransparent Thermal Barrier Coating," Journal of Thermophysics and Heat Transfer, Vol. 11. No. 2, 1997, pp. 315318.

'Siegel. R., "Two-Flux and Green's Function Method for Transient Radiative Transfer in a Semitransparent Layer," Proceedings of the Ist International Symposium on Radiative Heat Transfer (Kuşadasi, Turkey), Begell House, New York, 1996. pp. 473-487.

${ }^{\text {k}}$ Siegel. R., and Spuckler, C. M., "Approximate Solution Methods for Spectral Radiative Transfer in High Refractive Index Layers," International Journal of Heat and Mass Transfer, Vol. 37, Suppl. 1, 1994, pp. 403-413.

${ }^{9}$ Siegel, R., and Howell, J. R., Thermal Radiation Heat Transfer, 3rd ed., Hemisphere, Washington, DC, 1992, pp. 23 and 33.

${ }^{10}$ Viskanta. R., and Grosh, R. J., "Heat Transfer by Simultaneous Conduction and Radiation in an Absorbing Medium, "Journal of Heat Transfer, Vol. 84, No. 1, 1962, pp. 63-72. 